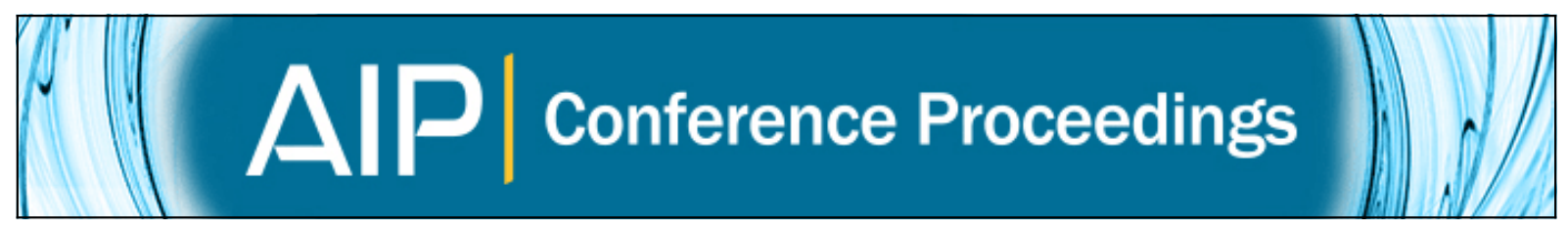

\title{
Study of Linear Magnetic Filters in a Pulsed Copper Vacuum Arc
}

L. Giuliani, D. Grondona, and H. Kelly

Citation: AIP Conference Proceedings 875, 199 (2006); doi: 10.1063/1.2405930

View online: http://dx.doi.org/10.1063/1.2405930

View Table of Contents: http://scitation.aip.org/content/aip/proceeding/aipcp/875?ver=pdfcov

Published by the AIP Publishing

\section{Articles you may be interested in}

On the mechanism of operation of a cathode spot cell in a vacuum arc

Appl. Phys. Lett. 104, 184101 (2014); 10.1063/1.4874628

Three-dimensional Ion Distribution in a Filtered Vacuum Arc Discharge

AIP Conf. Proc. 875, 254 (2006); 10.1063/1.2405943

Effects of pulsing parameters on production and distribution of macroparticles in cathodic vacuum arc deposition J. Vac. Sci. Technol. A 24, 957 (2006); 10.1116/1.2201046

Vacuum arc deposition devices

Rev. Sci. Instrum. 77, 021101 (2006); 10.1063/1.2169539

Mechanism for cathode spot grouping in vacuum arcs

Appl. Phys. Lett. 81, 3936 (2002); 10.1063/1.1523158 


\title{
Study of Linear Magnetic Filters in a Pulsed Copper Vacuum Arc
}

\author{
L. Giuliani*, D. Grondona** and H. Kelly ${ }^{* *}$ \\ R Instituto de Física del Plasma (CONICET), Departamento de Física, Facultad de Ciencias Exactas y \\ Naturales, (UBA) \\ Cdad. Universitaria, Pab. I, C1428EHA, Buenos Aires \\ ARGENTINA
}

\begin{abstract}
An experimental study of the plasma jet generated in a pulsed copper vacuum arc with an annular anode and operated with a linear magnetic filter is presented. Two types of filters were employed, one consisting of an insulating duct and the other of a conducting duct, both of them surrounded by an external coil, which generates the axial magnetic field. To improve the ion flux at the filter entrance another auxiliary magnetic field in the cathode region was applied (focusing field). Also, the cathode shape was modified to confine the cathode spot onto the front cathode surface. Operating the arc under vacuum conditions, probe measurements of the ion saturation current and the plasma potential at different axial positions along the ducts and for different filtering magnetic field values are reported. A comparison between both kinds of filters is also presented.
\end{abstract}

Keywords: Plasma, Vacuum arc, Magnetic filter

PACS: 52, 52.80.Vp, 52.77-j

\section{INTRODUCTION}

The ion transport through axial magnetic fields is of special interest for Vacuum Arc plasma sources. These devices are attractive for the deposition of thin films, since an intense jet of metallic plasma including relatively high-energy ions (15-120 eV, depending on the cathode material and charge-state of the ions) is produced with a high efficiency at the cathode surface. The ions come from minute points on the cathode known as "cathode spots". Also, macroparticles, which are tiny droplets of melting cathode material of diameter $0.1-100$ microns are emitted from the spots, so an amount of macroparticles can be found on the substrate [1]. For some applications, such as for optical films, this is an unwanted effect. Several filtering systems have been developed to reduce or eliminate the macroparticles from the arc plasma [2]. Presently, the magnetic filtering is a widely used method for removing the macroparticles. This kind of filter consists in a tube with an axial magnetic field. The plasma flux is guided through the tube by the magnetic field, whereas the massive macroparticles bounce or stick on the duct walls because they travel in almost straight lines due to their inertia. The most successful filter design, up to the moment, consists in a twister duct with an s-shape.

The main drawback of magnetic filtering is the reduction of the deposition rate, due to ions losses along the filter duct. The filter efficiency can be defined as the ratio between the number of ions at the filter entrance and the number of ions at the end of the tube. For that reason, it is very important to confine the cathode spots on the surface of the cathode faced to the filter entrance just to increase the ions injected in the filter. There are many methods to confine the spot. Two of the more common methods are: one of them is covering all but the desired surface with an insulating material and another method makes use of an axial magnetic field that passes through the cathode surface. The magnetic field influences the motion of the arc spot. The arc drifts in the direction of the acute angle formed between the magnetic field and the cathode surface. Therefore, the magnetic field can either be diverging at the end of a cylinder cathode, or the

${ }^{*}$ Fellow of the CONICET

${ }^{* *}$ Member of the CONICET 
cathode can have tapered sides while the field is uniform.

Once the plasma entered the filter, to increase the ion transmission along the duct, biasing the duct wall is one of the most popular methods for optimizing conducting filters, since a positive bias will reject ions from reaching the filter (electron are magnetized) thus decreasing the ion loses.

In a previous work [3] a non-conducting straight filter was employed to study the ion flux transmission from a pulsed vacuum arc. The main drawback of this kind of filter is the fact that it cannot be biased. In this work, we present measurements of the ion current at the filter entrance using different cathode configuration to confine the spot. Also, we present a study of the ion flux transmission along a straight filter with conducting walls in order to evaluate the improvements that can be achieved by biasing the conducting filter.

\section{EXPERIMENTAL SET-UP}

A scheme of the experimental apparatus is shown in Fig 1. The arc was pulsed for $35 \mathrm{~ms}$, with an arc peak current of $450 \mathrm{~A}$. It was produced by discharging an electrolytic capacitor bank with $\mathrm{C}=0.075 \mathrm{~F}$, connected to a series inductor-resistor $(\mathrm{L}=2 \mathrm{mH}, \mathrm{R}=$ $0.33 \Omega$ ), which critically damped the discharge. A mechanically controlled tungsten trigger rod ignited the arc. The arc was operated in a high vacuum of 0.01 $\mathrm{Pa}$.

A grounded copper cathode $(5 \mathrm{~cm}$ in length and 1 $\mathrm{cm}$ in diameter) was placed in front an annular anode with an aperture of $5 \mathrm{~cm}$ and a length of $2 \mathrm{~cm}$. The distance between the cathode surface and the closest plane of the anode was $1 \mathrm{~cm}$. At the end of the anode it was located the entrance of the magnetic duct. The magnetic field was established by an external coil wrapped around the tube, $23 \mathrm{~cm}$ long and with an inner wall radius of $5 \mathrm{~cm}$. The coil was fed with dc current from an independent power source. The magnetic field strength was measured with a calibrated Hall probe, and the magnetic field intensity $(B)$ was characterized with the value measured at the coil center. The maximum magnetic field that could be obtained was $293 \mathrm{G}$.

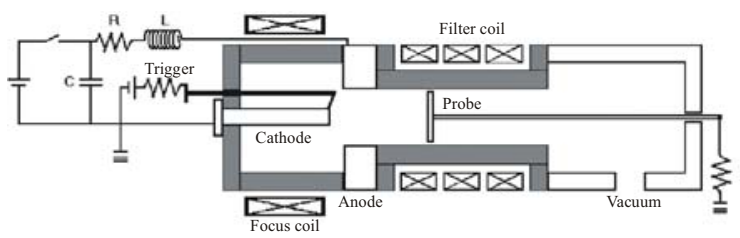

FIGURE 1.Schematic diagram of the experimental set-up
Three different cathode configurations were used to enhance the ion number at the filter entrance. In the first configuration the lateral cathode surface was covered with a Pyrex insulator. In the second configuration a focusing magnetic $\left(\mathrm{B}_{\text {focus }} \sim 250 \mathrm{G}\right)$ field was added using the previous geometry. In the third one, a tapered cathode with a focusing field was used (See Fig. 2). The ion current at the filter entrance was measured using a collector plate with a radius almost coincident with the duct inner radius, so it collected nearly all the charges entering the duct.

A circular flat probe (radius $1.5 \mathrm{~cm}$ ) was used to measure the ion current $\left(I_{i}\right)$ along the filter. It was connected to ground through a resistor. Also, the probe floating potential $\left(V_{f l}\right)$ was measured by connecting the probe to a high impedance resistor. Both $I_{i}$ and $V_{f l}$ were measured as functions of the axial distances $(d)$ from the cathode surface for different $B$ values. All these measurements were performed with the tapered cathode and without a focusing magnetic field.

The measurements of the ion current along the conducting filter were performed biasing the filter wall with an external power source, and with a floating filter. The filter floating potential $\left(F_{f l}\right)$ was measured as a function of $B$.

The arc voltage drop $\left(V_{a c}\right)$ and the discharge current $\left(I_{d}\right)$ as functions of $B$ were measured using a resistive voltage divider and a calibrated small-value $\left(810^{-3} \Omega\right)$ resistor, respectively.
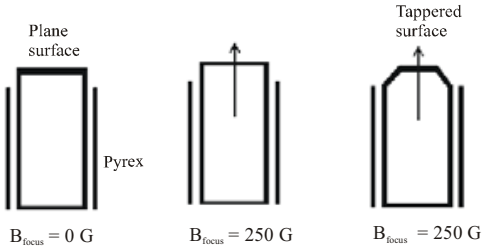

FIGURE 2: Different cathode configurations

\section{RESULTS}

The arc voltage and the discharge current were both independent of $B$, and took the values $V_{a c} \sim 45 \mathrm{~V}$ and $I_{d} \sim 450 \mathrm{~A}$.

The Ion current collected at the filter entrance for the cathode with the insulator shielding without a magnetic focusing field was $(2.0 \pm 0.1) \mathrm{A}$; with $\mathrm{B}_{\text {focus }} \sim$ $250 \mathrm{G}$ was $(3.1 \pm 0.1) \mathrm{A}$ and with the tapered cathode and with $\mathrm{B}_{\text {focus }} \sim 250 \mathrm{G}$ was $(3.6 \pm 0.1) \mathrm{A}$

The probe floating potential was almost independent of $B$ and $d$ with a value of $\sim 25 \mathrm{~V}$.

In Fig 3, the ion saturation current as function of $d$ with $B$ as a parameter is presented for the insulating filter. The same measurements for the conducting filter are shown in Fig. 4. 


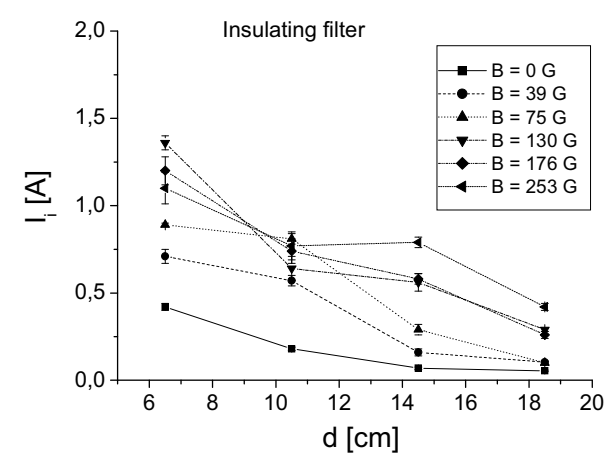

FIGURE 3. Ion current as a function of the cathode distance with the magnetic field at the filter as a parameter.

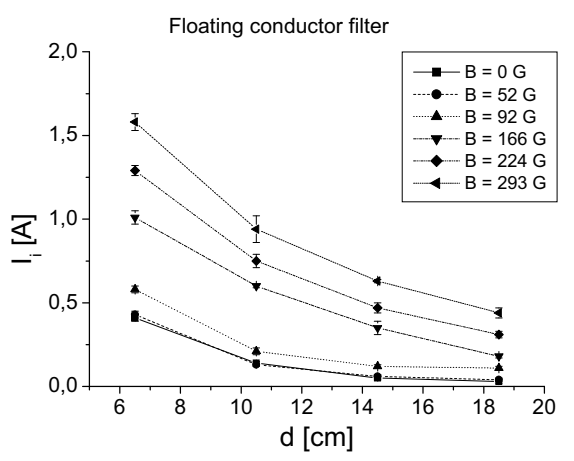

FIGURE 4. Ion current as a function of the cathode distance with the magnetic field at the filter as a parameter.

In a previous work [3] we found that for our device geometry the anodic sheath potential drop was about $10 \mathrm{~V}$, so considering that the plasma potential $\left(V_{p l}\right)$ can be obtained as the arc voltage $(\approx 45 \mathrm{~V})$ minus the anodic potencial drop, we found $V_{p l} \sim 35 \mathrm{~V}$.

In Fig. 5 the filter floating potential as a function of the filter magnetic field is shown. Note the horizontal line indicating the plasma potential in the figure.

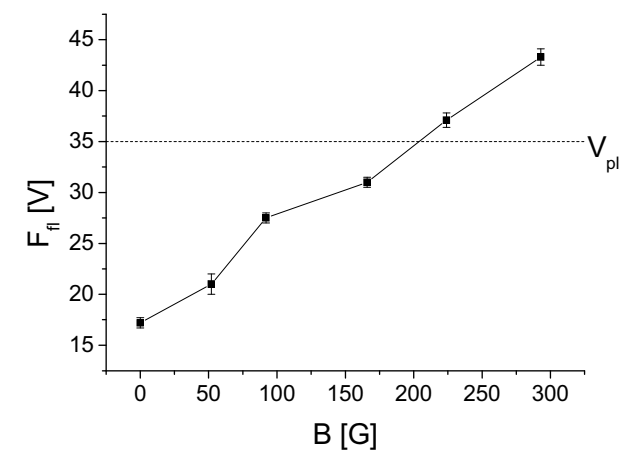

FIGURE 5. Filter floating potential as a function of the magnetic field at the filter.
In Fig. 6 and 7, the ion current collected with the probe at the filter exit as a function of the filter bias potential with the magnetic field of the filter as a parameter is presented.

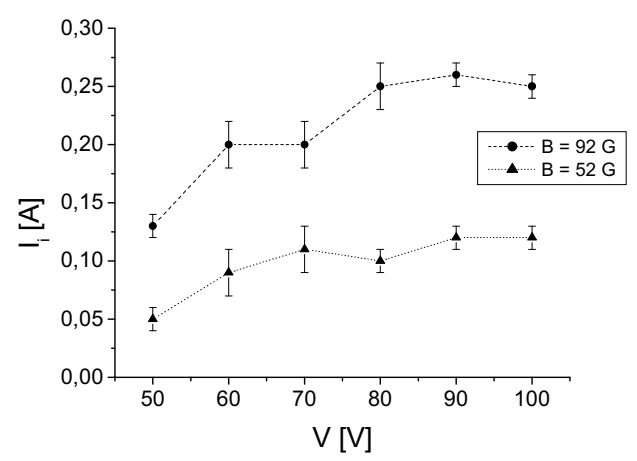

FIGURE 6. Ion current at the filter exit as a function of the filter bias potential with the magnetic field at the filter as a parameter.

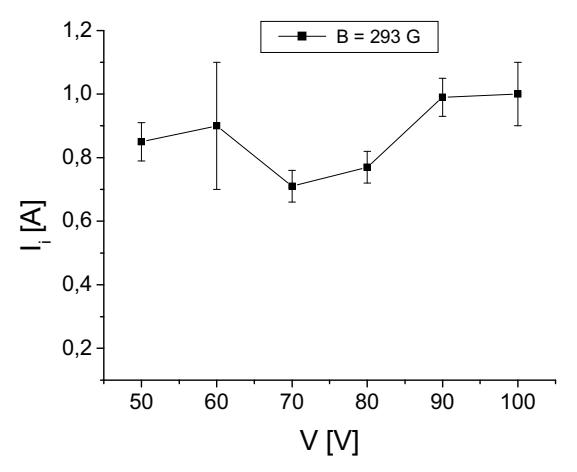

FIGURE 7. Ion current at the filter exit as a function of the filter bias potential with the magnetic field as the filter as a parameter.

\section{DISCUSSION}

From the measurements of the ion current at the filter entrance for the three different cathode configurations, it can be seen that the tapered cathode with a focusing magnetic field produces an increase of around $20 \%$ in $I_{i}$ with respect to the non-tapered shape with a focusing magnetic field and a $90 \%$ increase with respect to the plane surface without $B_{\text {focus }}$.

The ion flux profiles presented in figs. 3 and 4, insulating and floating conductor ducts, respectively, show no clear differences between both kinds of filters. This is a somewhat unexpected result, and can be attributed to a thin metallic coating grown on the inner wall of the insulating duct that produces an effective floating potential of this duct similar to that reached in the conductor duct. In both kinds of filters 
there is a strong dependence of $I_{i}$ on $B$ (for a fixed $d$ value): for instance, at the filter exit, the current increases by a factor of $\sim 10$ when comparing the values corresponding to $B=293 \mathrm{G}$ and $B=52 \mathrm{G}$.

The floating potential of the conducting filter as a function of $B$ presented in Fig. 5 shows an expected behavior. For low $B$ values it takes negative values with respect to the plasma potential, but for higher $B$ values it increases reaching values even higher than the plasma potential, as a consequence of the strong magnetization of the electrons.

The ion current measured at the filter exit as a function of the filter bias voltage for different $B$ values (Figs. 6 and 7) shows only a weak dependence of $I_{i}$ within experimental uncertainties (note that the biasing voltage values presented in the figures are well beyond $V_{p l}$, so the filter is rejecting ions). However, the same strong dependence of $I_{i}$ on $B$ found in the floating filter case is again found with a biased filter: for a bias voltage of $100 \mathrm{~V}$, the current is again increased in a factor of $\sim 10$ when comparing its values for $B=293 \mathrm{G}$ and $B=52 \mathrm{G}$.

\section{ACKNOWLEDGMENTS}

This work was supported by grants from the Agencia Nacional de Promoción científica y Tecnológica (PICT 03-09491), Universidad de Buenos Aires (PID X111, PID X146), CONICET (PIP 02239, PEI 6011).

\section{REFERENCES}

1. Boxman R.L., Sanders D.M. and Martin P.J., Handbook of Vacuum Arc Science and Technology, Fundamentals and Applications, Park Ridge, New Jersey: Noyes, (1995).

2. Krzysztof Miernik, Jan Walkowicz, Jan Bujak Plasma \& Ions 3, 41-51(2000).

3. D. Grondona, H. Kelly, L. Giuliani, Brazilian Journal of Physics, Vol. 34, pp. 1523 - 1526, (2004). 